Supporting Information:

\title{
Structural Basis for substrate specificity and carbapenemase activity of OXA-48 Class D $\beta$-lactamase
}

Afroza Akhtar†, Orville A. Pemberton†, Yu Chen†*

Department of Molecular Medicine, University of South Florida College of Medicine, 12901

Bruce B. Downs Blvd, MDC 3522, Tampa, Florida 33612, United States

*Corresponding author: Yu Chen, ychen1@usf.edu

\section{Table of Contents}

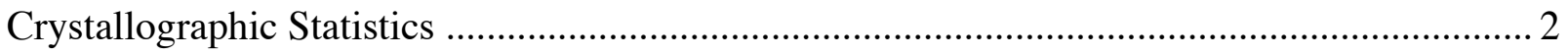

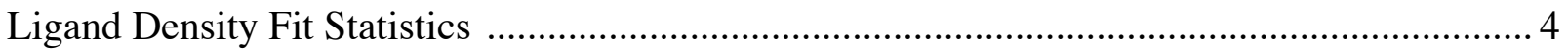

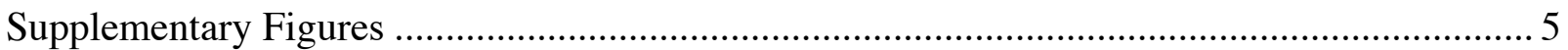


Supplementary Table S1. Crystallographic data collection and refinement statistics

\begin{tabular}{|c|c|c|c|c|}
\hline & $\begin{array}{c}\text { OXA-48 } \\
\text { Meropenem }\end{array}$ & $\begin{array}{c}\text { OXA-48 } \\
\text { Faropenem }\end{array}$ & $\begin{array}{l}\text { OXA-48 } \\
\text { Imipenem }\end{array}$ & $\begin{array}{c}\text { OXA-48 } \\
\text { Imipenem HP }\end{array}$ \\
\hline \multicolumn{5}{|l|}{ Data collection } \\
\hline Space group & $P 2{ }_{1}$ & $P 2{ }_{1}$ & $P 21$ & $P 2{ }_{1}$ \\
\hline \multicolumn{5}{|l|}{ Cell dimensions } \\
\hline$a, b, c(\AA)$ & $\begin{array}{c}59.79,106.51 \\
95.83\end{array}$ & $\begin{array}{c}59.55,106.67 \\
96.26\end{array}$ & $\begin{array}{c}\text { 58.49,106.66, } \\
94.66\end{array}$ & $\begin{array}{c}58.02,105.43, \\
93.57\end{array}$ \\
\hline$\alpha, \beta, \gamma\left({ }^{\circ}\right)$ & $\begin{array}{c}90.00,104.66 \\
90.00\end{array}$ & $\begin{array}{c}90.00,104.19 \\
90.00\end{array}$ & $\begin{array}{c}90.00107 .59 \\
90.00\end{array}$ & $\begin{array}{c}90.00,107.96 \\
90.00\end{array}$ \\
\hline Resolution (Å) & $\begin{array}{l}30.9-2.0 \\
(2.1-2.0)\end{array}$ & $\begin{array}{l}35.1-2.1 \\
(2.2-2.1)\end{array}$ & $\begin{array}{l}33.0-2.0 \\
(2.1-2.0)\end{array}$ & $\begin{array}{l}32.59-1.75 \\
(1.81-1.75)\end{array}$ \\
\hline$R_{\text {merge }}$ & $0.08(0.70)$ & $0.07(0.37)$ & $0.10(0.57)$ & $0.11(0.83)$ \\
\hline$R_{\text {meas }}$ & $0.09(0.83)$ & $0.09(0.45)$ & $0.16(0.78)$ & $0.13(1.00)$ \\
\hline$R_{\text {pim }}$ & $0.05(0.45)$ & $0.05(0.25)$ & $0.08(0.41)$ & $0.07(0.55)$ \\
\hline CC1/2(outer shell) & 0.762 & 0.920 & 0.639 & 0.608 \\
\hline$I / \sigma(I)$ & $17.4(2.1)$ & $17.5(2.2)$ & $10.6(2.1)$ & $11.1(2.1)$ \\
\hline Completeness (\%) & 99.60 (99.70) & $98.40(90.90)$ & $93.20(88.90)$ & $89.50(84.50)$ \\
\hline Redundancy & $3.6(3.4)$ & $3.5(2.7)$ & $3.7(3.6)$ & $6.1(5.8)$ \\
\hline \multicolumn{5}{|l|}{ Refinement } \\
\hline Resolution ( $(̊)$ & $30.9-2.0$ & $35.1-2.1$ & $33.0-2.0$ & $32.59-1.75$ \\
\hline No. reflections/free & 77973 (7477) & 64787 (5695) & $69067(6376)$ & 95925 (9021) \\
\hline $\begin{array}{l}\text { Reflections(refinem } \\
\text { ent/free) }\end{array}$ & 77881(1999) & 64673(1994) & 69051(1995) & 95912 (4846) \\
\hline$R_{\text {work }} / R_{\text {free }}$ & $0.187 / 0.231$ & $0.177 / 0.227$ & $0.170 / 0.218$ & $0.158 / 0.200$ \\
\hline \multicolumn{5}{|l|}{ No. of atoms } \\
\hline Protein & 8037 & 8085 & 8017 & 8033 \\
\hline Ligand & 106 & 78 & 82 & 125 \\
\hline Water & 665 & 306 & 893 & 856 \\
\hline \multicolumn{5}{|l|}{$B$-factors } \\
\hline Protein & 34.92 & 39.43 & 17.78 & 16.97 \\
\hline Ligand & 46.25 & 47.35 & 26.77 & 31.00 \\
\hline Water & 39.71 & 39.63 & 26.97 & 26.83 \\
\hline \multicolumn{5}{|l|}{ R.m.s. deviations } \\
\hline Bond lengths ( $\AA$ ) & 0.007 & 0.007 & 0.003 & 0.007 \\
\hline Bond angles $\left({ }^{\circ}\right)$ & 0.84 & 0.84 & 0.49 & 0.87 \\
\hline \multicolumn{5}{|l|}{ Ramachandran plot } \\
\hline Favored (\%) & 97.84 & 97.03 & 98.14 & 97.70 \\
\hline Allowed (\%) & 2.05 & 2.86 & 1.86 & 2.19 \\
\hline Outliers (\%) & 0.10 & 0.10 & 0.00 & 0.10 \\
\hline PDB code & 6PT1 & 6PSG & 6PTU & 6РКО \\
\hline
\end{tabular}




\begin{tabular}{|c|c|c|}
\hline & $\begin{array}{c}\text { OXA-48 } \\
\text { Cefotaxime }\end{array}$ & $\begin{array}{l}\text { OXA-48 } \\
\text { Cefoxitin }\end{array}$ \\
\hline \multicolumn{3}{|l|}{ Data collection } \\
\hline Space group & $P 2{ }_{1}$ & $P 2{ }_{1}$ \\
\hline \multicolumn{3}{|l|}{ Cell dimensions } \\
\hline$a, b, c(\AA ̊)$ & $\begin{array}{c}59.73,107.01 \\
96.19\end{array}$ & $\begin{array}{c}60.25,107.12 \\
96.11\end{array}$ \\
\hline$\alpha, \beta, \gamma\left({ }^{\circ}\right)$ & $\begin{array}{c}90.00,104.17 \\
90.00\end{array}$ & $\begin{array}{c}90.00,104.06, \\
90.00\end{array}$ \\
\hline Resolution (Å) & $\begin{array}{l}41.6-2.0 \\
(2.1-2.0)\end{array}$ & $\begin{array}{l}26.9-2.3 \\
(2.4-2.3)\end{array}$ \\
\hline$R$ merge & $0.07(0.35)$ & $0.06(0.24)$ \\
\hline$R_{\text {meas }}$ & $0.08(0.42)$ & $0.07(0.28)$ \\
\hline$R_{\text {pim }}$ & $0.04(0.22)$ & $0.04(0.15)$ \\
\hline CC1/2(Outer shell) & 0.921 & 0.948 \\
\hline$I / \sigma(I)$ & $21.6(3.5)$ & $22.33(4.2)$ \\
\hline Completeness (\%) & $99.00(91.00)$ & $97.80(91.70)$ \\
\hline Redundancy & $3.6(2.7)$ & $3.7(3.2)$ \\
\hline \multicolumn{3}{|l|}{ Refinement } \\
\hline Resolution ( $\AA$ ) & $41.6-2.0$ & $26.9-2.3$ \\
\hline No. reflections/free & 73449 (6532) & 51181 (4811) \\
\hline $\begin{array}{l}\text { Reflections(refinem } \\
\text { ent/free) }\end{array}$ & 73283(1995) & $51098(2022)$ \\
\hline$R_{\text {work }} / R_{\text {free }}$ & $0.173 / 0.205$ & $0.205 / 0.247$ \\
\hline \multicolumn{3}{|l|}{ No. of atoms } \\
\hline Protein & 8072 & 8053 \\
\hline Ligand & 110 & 102 \\
\hline Water & 264 & 122 \\
\hline \multicolumn{3}{|l|}{$B$-factors } \\
\hline Protein & 42.97 & 52.03 \\
\hline Ligand & 50.62 & 56.72 \\
\hline Water & 43.60 & 49.90 \\
\hline \multicolumn{3}{|l|}{ R.m.s. deviations } \\
\hline Bond lengths ( $\AA$ ) & 0.006 & 0.002 \\
\hline Bond angles $\left({ }^{\circ}\right)$ & 0.78 & 0.51 \\
\hline \multicolumn{3}{|l|}{ Ramachandran Plot } \\
\hline Favored (\%) & 97.34 & 97.84 \\
\hline Allowed (\%) & 2.46 & 2.16 \\
\hline Outliers (\%) & 0.20 & 0.00 \\
\hline PDB Code & 6PQI & 6РT5 \\
\hline
\end{tabular}

*Data were collected from a single crystal for each structure. Values in parentheses are for highest-resolution shell. 
Supplementary Table S2. Ligand Density Fit Statistics

\begin{tabular}{|c|c|c|c|c|c|c|}
\hline & $\begin{array}{c}\text { OXA-48 } \\
\text { Meropenem }\end{array}$ & $\begin{array}{c}\text { OXA-48 } \\
\text { Faropenem }\end{array}$ & $\begin{array}{c}\text { OXA-48 } \\
\text { Imipenem }\end{array}$ & $\begin{array}{c}\text { OXA-48 } \\
\text { Imipenem HP }\end{array}$ & $\begin{array}{c}\text { OXA-48 } \\
\text { Cefotaxime }\end{array}$ & $\begin{array}{l}\text { OXA-4 } \\
\text { Cefoxit }\end{array}$ \\
\hline \multicolumn{7}{|c|}{ RSR value } \\
\hline Chain A & 0.16 & 0.10 & 0.12 & 0.12 & 0.20 & 0.19 \\
\hline Chain B & 0.20 & 0.10 & 0.13 & 0.14 & 0.17 & 0.19 \\
\hline Chain C & 0.14 & 0.11 & 0.13 & 0.12 & 0.21 & 0.17 \\
\hline Chain D & 0.18 & 0.13 & 0.14 & 0.16 & 0.17 & 0.22 \\
\hline \multicolumn{7}{|l|}{ RSCC } \\
\hline Chain A & 0.85 & 0.97 & 0.91 & 0.91 & 0.84 & 0.90 \\
\hline Chain B & 0.87 & 0.96 & 0.91 & 0.91 & 0.82 & 0.87 \\
\hline Chain C & 0.89 & 0.94 & 0.90 & 0.92 & 0.85 & 0.88 \\
\hline \multicolumn{7}{|c|}{$\begin{array}{l}\text { Occupancy of } \\
\text { ligand }\end{array}$} \\
\hline Chain A & 0.90 & 0.95 & 0.86 & 0.85 & 0.81 & 0.85 \\
\hline Chain B & 0.86 & 0.94 & 0.80 & 0.80 & 0.91 & 0.88 \\
\hline Chain C & 0.85 & 0.97 & 0.85 & 0.80 & 0.84 & 0.86 \\
\hline Chain D & 0.94 & 0.96 & 0.80 & 0.78 & 0.92 & 0.89 \\
\hline
\end{tabular}




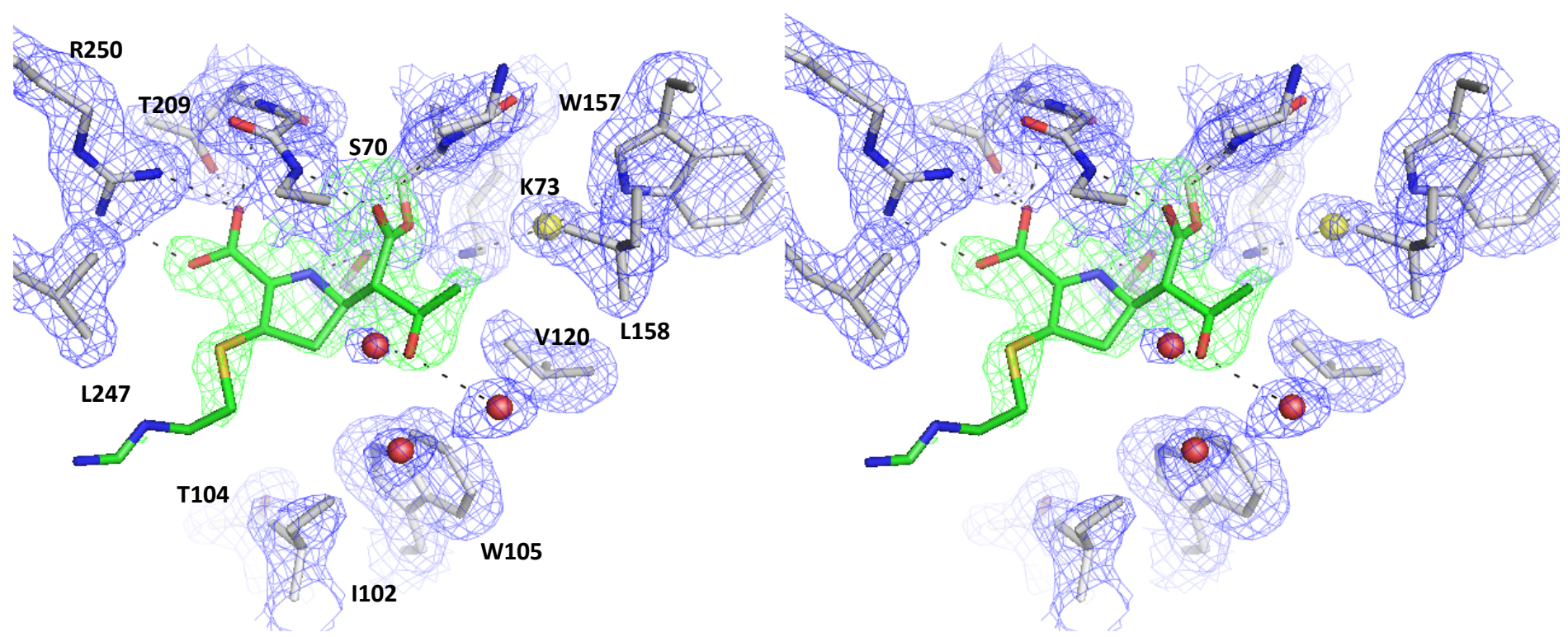

Figure S1. Stereo view of OXA-48:imipenem acyl-enzyme complex. The protein and ligand of the complex are shown in white and green, respectively. Hydrogen bonds are shown as black dashed lines. Chloride ion and water molecules are shown as yellow and red spheres respectively. The unbiased Fo-Fc electron density map around imipenem is shown at $3 \sigma$ (green). 2Fo-Fc map surrounding the active site residues is shown at $1.5 \sigma$ (blue).

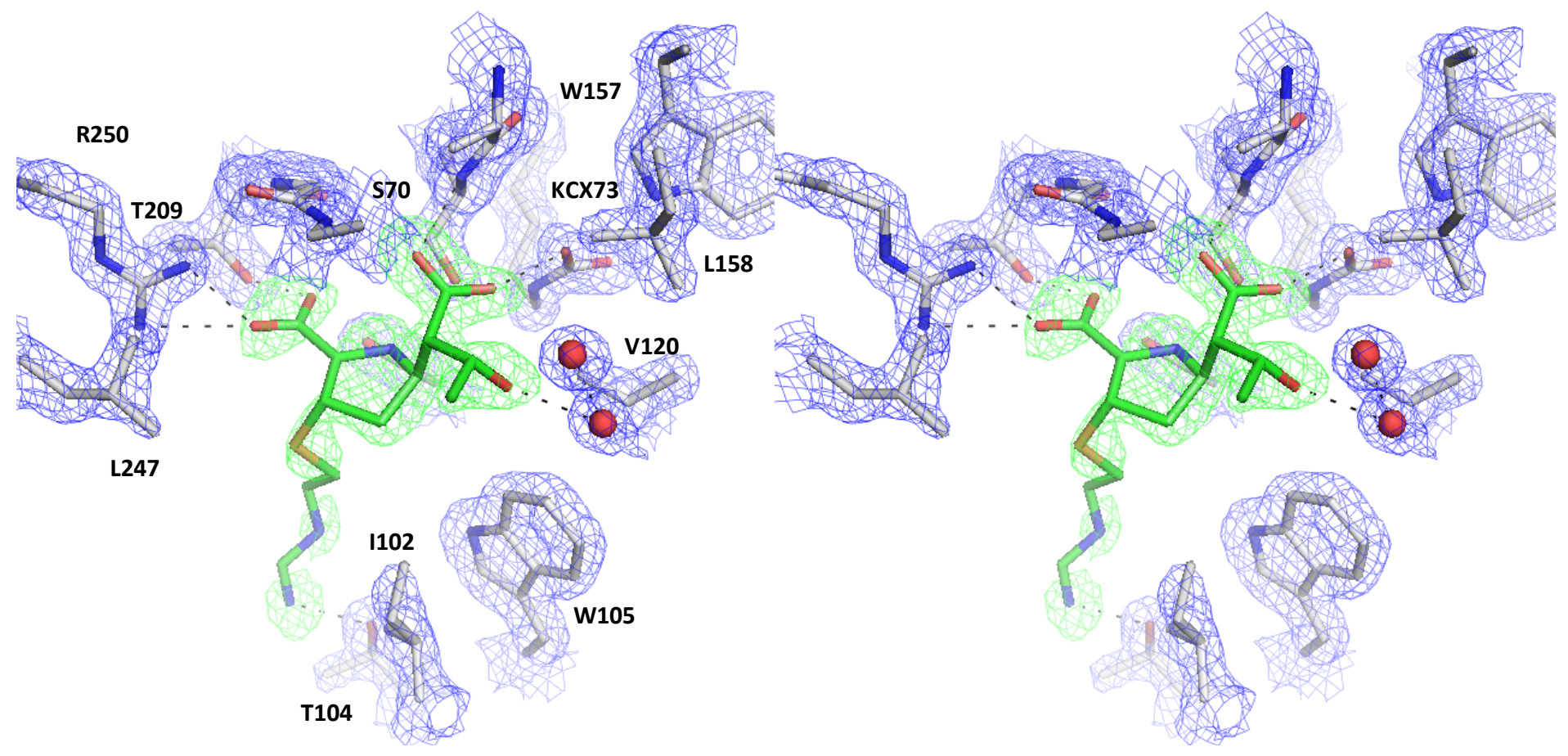

Figure S2. Stereo view of OXA-48 complex with hydrolyzed imipenem. The protein and ligand of the complex are shown in white and green, respectively. Hydrogen bonds are shown as black dashed lines. Water molecules are shown as red spheres. The unbiased Fo-Fc electron density map around imipenem is shown at $3 \sigma$ (green). 2Fo-Fc map surrounding the active site residues is shown at $1.5 \sigma$ (blue). 